\title{
Bilateral Morgagni Hernia: Primary Repair without a Mesh
}

\author{
Vassilios Papanikolaou Dimitrios Giakoustidis \\ Paraskevi Margari Nikolaos Ouzounidis \\ Nikolaos Antoniadis Alexander Giakoustidis \\ Dimitrios Kardasis Dimitrios Takoudas
}

Department of Transplant Surgery, Medical School, Aristotle University, Hippokration Hospital, Thessaloniki, Greece

\section{Key Words}

Bilateral Morgagni hernia - Transabdominal approach · Mesh

\begin{abstract}
We present a case of bilateral Morgagni hernia in a 68-year-old male with an intermittent history of progressive onset of breath shortness and occasional cardiac arrhythmias. Diagnosis was made by clinical examination and the findings in a plain chest radiograph and was confirmed by computed tomography scan. The patient was operated electively and subjected to a transabdominal approach. A bilateral subcostal incision revealed a large right side anterior diaphragmatic defect with a hernia containing the ascending colon, the majority of the transverse colon and a huge amount of omentum. Also a second smaller defect was found on the left side with no hernia inside. After large bowel and omentum had been taken down to the peritoneal cavity, both defects were primarily closed using interrupted nylon sutures without the use of a mesh. The patient recovered very well, had an uneventful postoperative course and was released on the 5 th postoperative day. 15-month follow-up failed to reveal any signs of recurrence.
\end{abstract}

\section{Introduction}

Morgagni hernias result from failure of the septum transversum to fuse with the thoracic wall and occur between the sternal and costal diaphragmatic attachments in the anterior mediastinum. They are the least common of the four types of congenital diaphragmatic hernias and account for $2-3 \%$ of all cases $[1,2]$. When they are discovered in adults, symptoms are often mild or absent. They may cause a number of potential complications, the latter usually being obstruction, bowel strangulation and volvulus, and if they are symptomatic they can cause a certain amount of distress to the patient. Most Morgagni hernias are discovered on chest radiograph or upper gastrointestinal study 
performed because of non-specific complaints, or they are diagnosed incidentally when the patients are being investigated for unrelated problems. Repair of the defect is indicated in all cases because of the risk of viscera obstruction or incarceration. Reports in the literature describe repair of the abnormality by the transabdominal or transthoracic approach with or without a mesh [3-5]. Since the last decade minimally invasive techniques have also been applied in the management of Morgagni hernias [6], and laparoscopic repair with the use of a mesh is now widely applicable.

Our report presents an interesting case of bilateral Morgagni hernia in an elderly patient with a history of progressive onset of shortness of breath and occasional cardiac arrhythmias.

\section{Case Report}

Our case is a 68-year-old male patient working as a farmer, who was admitted to a peripheral hospital for elective hydrocele repair. The routine plain chest X-ray revealed pleural effusion and the patient was referred to our department for further investigation. He reported an intermittent history of breath shortness and occasional cardiac arrhythmias, especially after hard work. On physical examination blood pressure and heart rate were normal, but respiratory sounds were found to be diminished at the right basal region on auscultation. The abdomen was soft, nondistended, and nontender. The right liver appeared to be normal, but percussion on its left lobe revealed flatulence, indicating the presence of bowel. The diagnosis of Morgagni hernia was made and confirmed by chest computed tomography scan. Bowel loops were herniated into the right thoracic cavity through a right side anterior diaphragmatic defect (fig. 1). The patient was operated electively and subjected to a transabdominal approach through a bilateral subcostal incision. There was a right anterior diaphragmatic hernia approximately $10 \mathrm{~cm}$ in diameter with the ascending colon, the majority of the transverse colon and a huge amount of omentum, all within the hernia sac (ig. 2). The hernia extended approximately one half of the way up to the apex of the right lung. There was a second defect of approximately $4 \mathrm{~cm}$, but with no hernia, in the left side diaphragm. The intestines were reduced into the abdomen with gentle traction (fig. 3 ) and the hernia sac was easily mobilised into the peritoneal cavity and excised. The defects were closed with nonabsorbable interrupted mattress sutures (No. 1 Prolene, Ethicon, USA), between the posterior edge of the defect and the posterior rectus sheath (fig. 4 ). There was no tension on the repair and there was no need for mesh implantation on either side. No drains were placed, the postoperative course was uneventful and the patient was discharged on the 5th postoperative day.

\section{Discussion}

Four types of congenital diaphragmatic hernias have been described: hiatal hernia with a defect at the esophageal hiatus, paraesophageal hernia with a defect adjacent to the hiatus, Bochdalek hernia with a defect posterolaterally, and Morgagni hernia with the defect being anteromedial or retrosternal. Each type requires its own closing technique, with prosthetic reinforcement often needed for larger defects $[7,8]$.

In 1769, Morgagni described for the first time a diaphragmatic hernia that originated from the sternocostal trigone. About $90 \%$ of Morgagni hernias are located on the right side, with $2 \%$ located on the left and $8 \%$ bilateral $[9,10]$. Morgagni hernias usually remain asymptomatic in infancy and childhood, but these hernias may progressively enlarge and become symptomatic, especially in adulthood. Exercise and excessive work can cause symptoms which are related to dyspnoea. Patients may also show signs and symptoms of acute incarceration or strangulation if their abdominal contents become trapped into the hernia sac. Our patient was mostly asymptomatic with an intermittent history of progressive onset of shortness of breath and occasional cardiac arrhythmias, especially after excessive work. 
The diagnosis of Morgagni hernia is usually made radiographically. Depending on the contents of the hernia, omentum, stomach, small intestine, or liver, it can appear differently on chest radiography and the diagnosis can be missed. Differential diagnosis should be made between intrathoracic tumour, atelectasis, pneumonia, or pericardial cyst. This may affect the decision to operate and the type of operation carried out, those being the transabdominal and the transthoracic approach. Contrast examination carried out for gastrointestinal symptoms can be perfectly normal, but computed tomography scan can be considered to be an accurate, noninvasive method of diagnosing Morgagni hernia. Moreover it can establish a diagnosis if the hernia sac is empty or contains omentum or part of the liver. Magnetic resonance imaging has been also used as a noninvasive modality to distinguish Morgagni hernia from other mediastinal masses [11, 12]. In our case, diagnosis was made by physical examination and plain chest radiogram and confirmed by computed tomography. Computed tomography showed a large diaphragmatic hernia with air-filled colon and omentum in the right chest cavity.

Although the majority of these hernias are asymptomatic, repair is recommended to avoid future complications. Primary repair can be done by transabdominal or transthoracic approach. The thoracic approach is preferably used in cases where there is a question as to Morgagni hernia and other thoracic abnormalities, such as a mediastinal cyst or mass or a bronchogenic carcinoma [12-14], although with the advances in preoperative imaging the technique is less frequently applied. The transabdominal approach is favoured when the diagnosis is certain, as it allows easier reduction of the hernia, especially for bilateral hernias. Furthermore, abdominal viscera within the hernia can be easily pulled down to their normal location into the abdomen $[4,5]$. Since the introduction of the laparoscopic approach, the method is increasingly applicable. The hernia sac can be viewed through the laparoscope, and the contents can be easily reduced once the peritoneum at the perimeter of the defect is incised. The sac is usually not removed and the defect is closed with sutures and reinforced with a mesh stapled onto the diaphragm [6]. In our patient we preferred the transabdominal approach, and a bilateral subcostal incision was made. The reason for this decision was the accuracy of the preoperative diagnosis and the existence of a large defect.

Follow-up after operative repair can be done with a chest radiograph and computed tomography. As of this writing, we have follow up our patient with chest radiography and computed tomography for 15 months so far with no sign of recurrence. 
Fig. 1. CT scan of the lungs and abdomen showing part of the bowel and omentum in the chest.

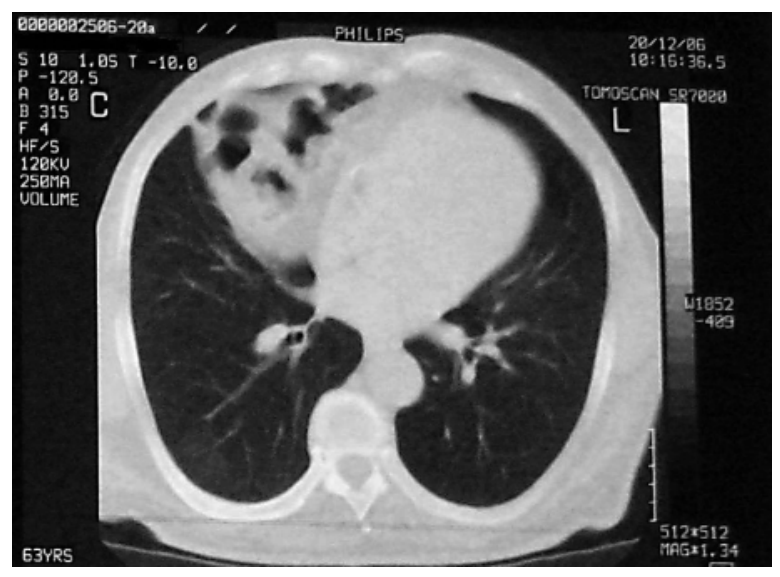

Fig. 2. Transabdominal approach through a bilateral subcostal incision, showing part of the large bowel and omentun inside the right defect. The left defect is not shown.

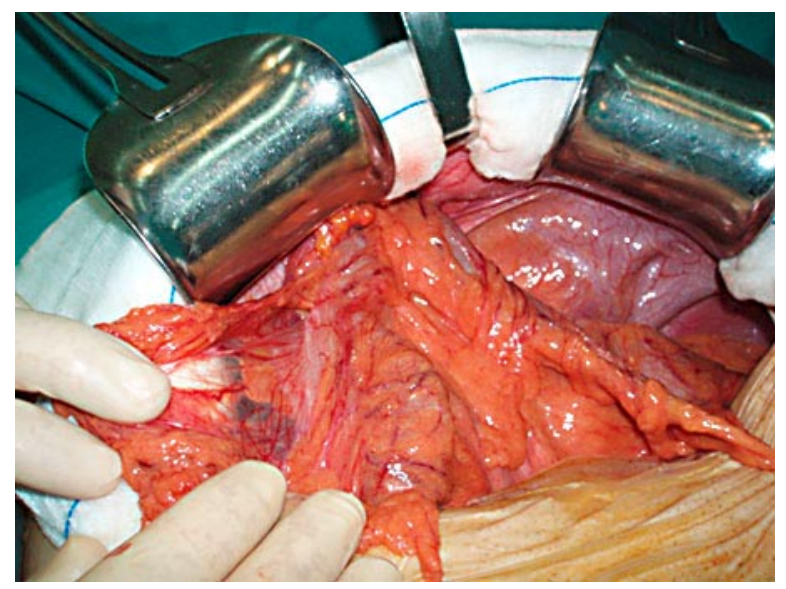

Fig. 3. Intraoperative photograph showing the right large defect after large bowel and omentum was taken down.

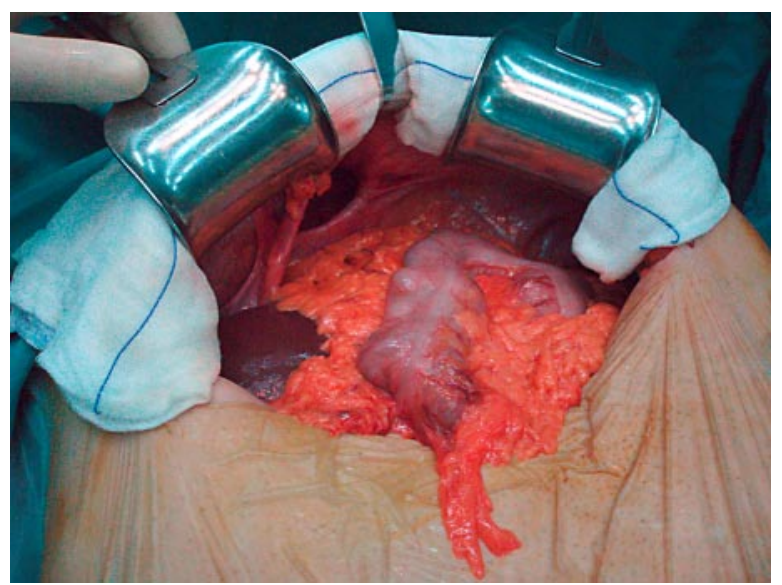


Fig. 4. Final repair of both defects after excision of the right hernia sac with interrupted nylon sutures.

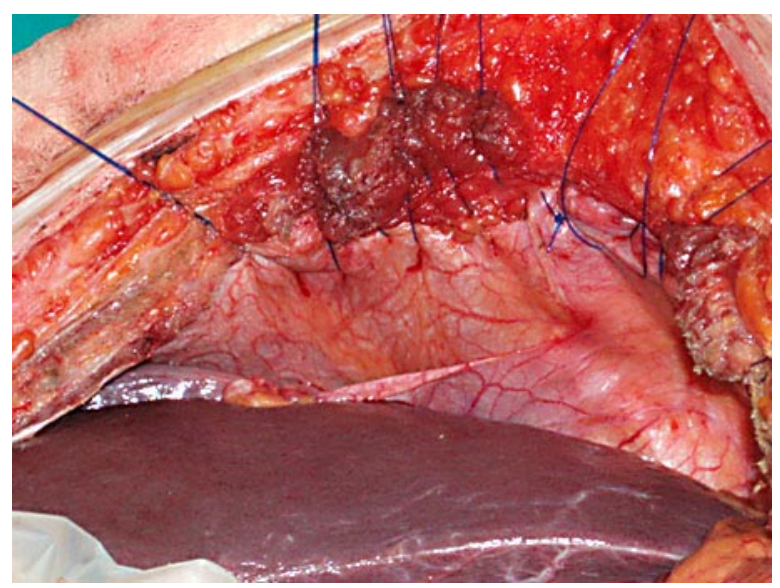




\section{References}

1 Harrington SW: Clinical manifestations and surgical treatment of congenital types of diaphragmatic hernia. Rev Gastroenterol 1951;18:243-256.

- Comer TP, Clagett OT: Surgical treatment of hernia of the foramen of Morgagni. J Thorac Cardiovasc Surg 1966;52:461-468.

-3 Paris F, Tarazona V, Casillas M, Blasco E, Canto A, Pastor J, Acosta A: Hernia of Morgagni. Thorax 1973;28:631-636.

4 Chin EF, Duchesne ER: The parasternal defect. Thorax 1955;10:214-219.

5 Kilic D, Nadir A, Doner E, Kavukcu S, Akal M, Ozdemir N, Akay H, Okten I: Transthoracic approach in surgical management of Morgagni hernia. Eur J Cardiothorac Surg 2001;20:1016-1019.

6 Kuster GG, Kline LE, Garzo G: Diaphragmatic hernia through the foramen of Morgagni: laparoscopic repair case report. J Laparoendosc Surg 1992;2:93-100.

7 Hartman GE: Diaphragmatic hernia; in Behrman RE, Kliegman RM, Arvin AM (eds): Nelson Textbook of Pediatrics, ed 5. Philadelphia, Saunders, 1996, pp 11611164 .

8 Yamashita K, Tsunoda T: Three-dimensional computer images of Morgagni hernia. Am J Surg 2003;187:109-110.

9 Federico JA, Ponn RB: Foramen of Morgagni hernia; in Shields TW, LoCicero J III, Ponn RB (eds): General Thoracic Surgery, ed 5. Philadelphia, Lippincott Williams and Wilkins, 2000, pp 647-660.

10 Hussong RL, Landreneau RJ, Cole FH: Diagnosis and repair of a Morgagni hernia with video-assisted thoracic surgery. Ann Thorac Surg 1997;63:1474-1475.

11 Kamiya N, Yokoi K, Miyazawa N, Hishinuma S, Ogata Y, Katayama N: Morgagni hernia diagnosed by MRI. Surg Today 1996;26:446-448.

12 Fagelman D, Caridi JG: CT diagnosis of hernia of Morgagni. Gastrointest Radiol 1984;9:153-155.

13 Lin ST, Moss DM, Henderson SO: A case of Morgagni hernia presenting as pneumonia. J Emerg Med 1997;15:297-301.

14 Al-Salem AH, Nawaz A, Matta H, Jacobsz A: Herniation through the foramen of Morgagni: early diagnosis and treatment. Pediatr Surg Int 2002;18:93-97. 